\title{
BER Analysis and Specifications of Future Generation Coalescing 5G and Cognitive Radio
}

\author{
Bhuvnesh Arora ${ }^{1}$ and Pallvi Chawla ${ }^{2}$ \\ Department of ECE \\ 1Jaypee Institute of Information Technology \\ Noida, Uttar Pradesh, India- 201307 \\ ${ }^{2}$ Jaypee University of Information Technology \\ Waknaghat, Solan, H.P., India- 173234
}

\author{
Reema Budhiraja \\ Department of Electronics and Communication \\ Engineering \\ Jaypee Institute of Information Technology \\ Sector-62, Noida, Uttar Pradesh, \\ India- 201307
}

\begin{abstract}
The intention of coalescing $5 \mathrm{G}$ and cognitive radio is to combine the information from wireless networks, portray and grow new technologies, networks and systems that assimilate into a flexible and dynamically-operating architecture. This paper is paying attention on the specifications of future generations and latest technologies which will be used in upcoming wireless mobile communication networks. This combination of technologies has been used to coerce the better integration, adaptation, modularity and standardization. Specially, the cross layer approach is used to surpass more information across layers. Radio access technology procedures enable higher peak data rates for end users as well as higher capacity and wider coverage.
\end{abstract}

\section{Keywords}

Fifth generations (5G), Cognitive Radio (CR), Wireless Innovative System for Dynamic Operating Mega communications (WISDOM), Cross Layer, Quality of Service (QoS), Radio Access Technology (RAT)

\section{INTRODUCTION}

Wireless with intelligence [1]-[2] will be encapsulated in mortal environment. Organization, home, public places, will create a new platform that enables omnipresent sensing, figure out stock house and communication. Major requirements for this kind of omnipresent ambient intelligence are that the devices are autonomous, accurate and robust. They can be deployed easily and need little maintenance. Mobile device [3] will be the arch to personally access ambient intelligence and needed information. These types of devices also imply micro/macro size and restrictions on the power handling. Logical connectivity with other devices and rigid networks is a compelling enabler for ambient intelligence system - this leads to very high data rates \& Quality of Service and service applications with space roaming [1]-[5].

5G technology [6]-[7] refers to short name of fifth Generation which was started from late 2010s. 5G has complete wireless communication with almost no limitations. It is highly supportable to WWWW (Wireless World Wide Web).

In the most challenging OSA (Open systems) architecture for which an embodiment will be new generation of radio systems called "Cognitive Radio" (CR) [8]-[9]. In this case secondary users, sharing spectrum [10]-[14], perform opportunistically seeking out unoccupied spectrum ("Spectrum Holes") and, at the same time, avoiding interference to primary users.
In this paper, the combination of $5 \mathrm{G} \& \mathrm{CR}$ has been proposed to bring together the existing wireless and wired communication techniques into an all IP (Internet Protocol) high performance worldwide network and communication without limitation, which bring us perfect real world wireless - WWWW. From this combination, we achieved unlimited wireless world interconnection, convergence and cooperation with a large variety of multimedia services and good spectral efficiency. In this paper, the performance of $5 \mathrm{G}$ and cognitive radio is analyzed on the basis of data rate, coverage and spectral efficiency.

Rest of the paper is organized as follows. Section 2 provides an overview of advancement in technology and progression in knowledge or machinery. In Section 3, performance of $5 \mathrm{G}$ and cognitive radio is analyzed on the basis of data rate, coverage and spectral efficiency. Section 4 provides an impression of future technology that coalesce $5 \mathrm{G}$ and cognitive radio. In Section 5, simulation results are plotted on account of data rate and spectrum utilization and some conclusions are drawn in Section 5.

\section{EVOLUTION OF TECHNOLOGY}

Technology from manufacturers is advancing at a stunning rate and the wireless networking is tying our gadgets together with the services we demand. In the past 10 years, an astonishing evolution of wireless services has seen which are used every day. With the exponential evolution, there has been equally exponential growth in use of the services, taking advantage of the recently available bandwidth around the world [1]-[5]. Table I shows the different generations with their data rates and associated technology. 
Table I. Evolution of Mobile Technology

\begin{tabular}{|c|c|c|c|}
\hline Generation & System & Date Rate & Technology Used \\
\hline \multirow{4}{*}{$1 \mathrm{G}$} & AMPS & \multirow{4}{*}{$\sim 1-5 \mathrm{Kbps}$} & \multirow{4}{*}{$\begin{array}{c}\text { Analog } \\
\text { Technology }\end{array}$} \\
\hline & TACS & & \\
\hline & NMT & & \\
\hline & J-TACS & & \\
\hline \multirow[b]{2}{*}{$2 \mathrm{G}$} & GSM & \multirow[b]{2}{*}{$10 \mathrm{Kbps}$} & \multirow{2}{*}{$\begin{array}{c}\text { Based on } \\
\text { TDMA \& FDMA }\end{array}$} \\
\hline & CDMA & & \\
\hline \multirow[b]{2}{*}{$2.5 \mathrm{G} / 2.75 \mathrm{G}$} & GPRS & $\sim 50 \mathrm{Kbps}$ & \multirow{2}{*}{$\begin{array}{c}\text { Based on } \\
\text { TDMA \& FDMA }\end{array}$} \\
\hline & EDGE & $\sim 200 \mathrm{Kbps}$ & \\
\hline \multirow[b]{2}{*}{$3 \mathrm{G}$} & WCDMA/UMTS & \multirow[b]{2}{*}{$384 \mathrm{Kbps}$} & \multirow[b]{2}{*}{ CDMA Technique } \\
\hline & CDMA 2000 & & \\
\hline \multirow[b]{2}{*}{$3.5 \mathrm{G}$} & HSDPA/HSUPA & \multirow[b]{2}{*}{ 5-30 Mbps } & \multirow[b]{2}{*}{ CDMA Technique } \\
\hline & $\begin{array}{l}\text { 1X EVDO } \\
\text { Rev. A,B,C }\end{array}$ & & \\
\hline \multirow[b]{2}{*}{$3.9 \mathrm{G} / 4 \mathrm{G}$} & LTE & \multirow[b]{2}{*}{$100-200 \mathrm{Mbps}$} & \multirow{2}{*}{$\begin{array}{l}\text { OFDMA \& } \\
\text { SCFDMA }\end{array}$} \\
\hline & WIMAX & & \\
\hline Beyond 4G & LTE-Advanced & Up to $1 \mathrm{Gbps}$ & $\begin{array}{l}\text { Hybrid of } \\
\text { OFDMA \& } \\
\text { SCFDMA }\end{array}$ \\
\hline
\end{tabular}

The First generation of wireless telecommunication technology is known as $1 \mathrm{G}$, was introduced in 1980. It consists Advance Mobile Phone Service (AMPS), Nordic Mobile Telephone (NMT) and Total Access Communication System (TACS). AMPS was 1st launched in USA in $1 \mathrm{G}$ mobile systems.

$2 \mathrm{G}$ technology refers to the 2 nd generation which is based on GSM. It was launched in Finland in year 1991. 2G network uses digital signals. Its data speed was up to $64 \mathrm{kbps}$. It enables services such as text messages, picture messages and MMS (multimedia message). It provides better quality and capacity.

$2.5 \mathrm{G}$ is a technology between the second $(2 \mathrm{G})$ and third generation of mobile telephony. $2.5 \mathrm{G}$ is sometimes described as $2 \mathrm{G}$ cellular technology combined with GPRS. Features includes in this $2.5 \mathrm{G}$, are phone calls, send receive E-mail messages, web browsing, speed: 64-144 kbps, camera phones, It takes time of 6-9 minutes to download a Mp3 song.

$3 \mathrm{G}$ technology refers to the third generation which was introduced in year 2000s. In this generation, data transmission increased from 144kbps- $2 \mathrm{Mbps}$. Phones of this generation, are specifically called smart phones and the features increase its bandwidth and data transfer rates, to accommodate web based applications, audio and video files.

4G technology [5] was started from late 2000s. Capable of providing $100 \mathrm{Mbps}-1 \mathrm{Gbps}$ speed. One of the basic term is used to describe is $4 \mathrm{G}$ is MAGIC.

\section{MAGIC:-}

- Mobile Multimedia

- Anytime Anywhere

- Global Mobility Support

- Integrated wireless Solution

- Customized Personal Services
Some features of $4 \mathrm{G}$ include more security, high speed, high capacity, low cost per-bit etc. Drawbacks of $4 \mathrm{G}$ are more battery usage, hard to implement, need complicated hardware, expensive equipment required to implement next generation network. Here are the 10 countries with the largest percentages of LTE subscriptions per capita projected for 2013 .

\section{CONCEPTUAL DESIGN OF 5G AND COGNITIVE RADIO}

\subsection{G Technology-}

5G technology [6] refers to short name of fifth Generation which was started from late 2010s. $5 \mathrm{G}$ endows with complete wireless communication with almost no limitations. It is highly supportable to WWWW (Wireless World Wide Web). 5G technology offers broadcasting of data in Gbps, multimedia services at higher data rate, watch T.V programs with clarity as to that of an HD Quality. Large phone memory, dialing speed, clarity in audio/video, interactive multimedia services, voice, streaming video, Internet and other services, make $5 \mathrm{G}$ more effective and attractive.

5G world has some technologies and objectives [7], which are shown in figure 1 and described below-

Powerful infrastructures

- Small cells/ Heterogeneous Networks

- Multi-technology (mobile, Wi-Fi)

- Device-to-Device

- Machine-to-Machine

- Cognitive Radio Networks

- Diverse spectrum bands

- Multi-objective context (traffic, mobility, radio condition) handling

- $\quad$ QoE (carrier grade, usability)

- $\quad$ Spectrum efficiency (licensed, unlicensed, shared)

- Resource use efficiency: computing, storage, communication

- $\quad$ Energy efficiency

- Overall cost efficiency

\subsection{CR Technology-}

In the most challenging OSA (Open systems) architecture for which an embodiment will be new generation of radio systems called "Cognitive Radio" (CR) [8] - basic principle of acting can be called "Cause - No - Harm". The performance of $5 \mathrm{G}$ and cognitive radio is analyzed on the basis of data rate, coverage and spectral efficiency. In this case secondary users, sharing spectrum, perform opportunistically seeking out unoccupied spectrum ("Spectrum Holes") [9]-[10] and, at the same time, avoiding interference to primary users. Cognitive radio has cooperative opportunistic frequency selection with spectrum-sensing capability [13]. It is an enabling technology for faster deployment and increased spatial reuse. In the overlay CROFDM [11]-[14] system, using time domain hierarchical transmission or frequency domain hierarchical modulation, it is possible to have two concurrent transmission in given interference regions. At the receiver side, the first layer signal 
is decoded first and then cancelled from the overall received signal to achieve a clear acquisition of the second layer signal [11].
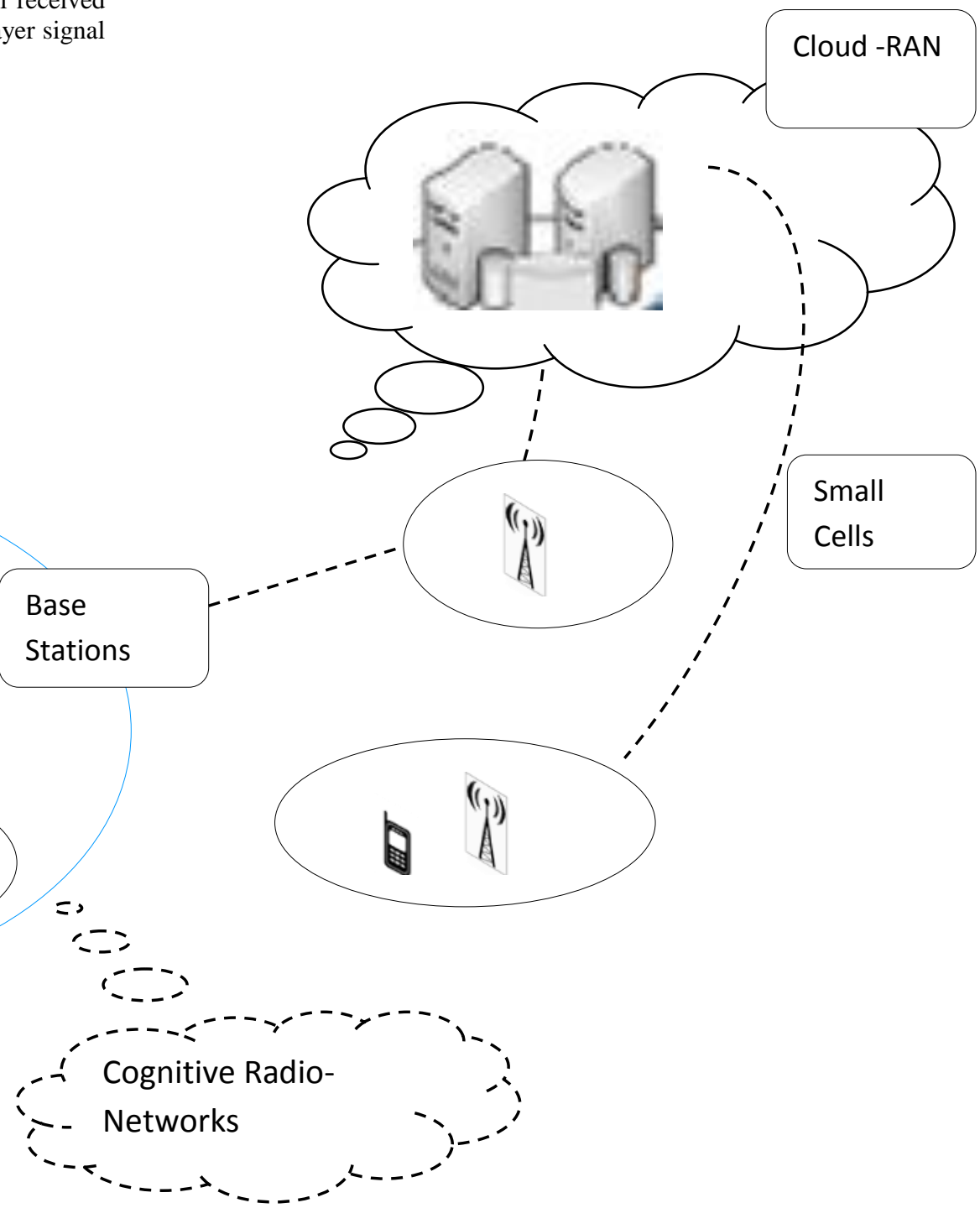

Figure 1: Infrastructure of 5G technology and objectives

\section{FUTURE TECHNOLOGY COALESCING 5G AND COGNITIVE \\ RADIO}

The Coalescing of $5 \mathrm{G}$ and $\mathrm{CR}$ plays a vital role in field of communications. The $\mathrm{CR}$ offers better possibility to connect in a functional way with the existing wireless technologies and, thus it offers a complete integrated $5 \mathrm{G}$ wireless access network.

The CR-5G network is strongly sustained by the main $5 \mathrm{G}$ requirements achievement through the $\mathrm{CR}$ use, in a highperformance manner-

- A 5G acquires the desirable terminal, that is, $\mathrm{CR}$ terminal.

- A 5G obtains the "tool" technology to interconnect and integrate all the wireless networks types, and thus, gains the needed network infrastructure.
- $\quad 5 \mathrm{G}$ adopts the already proposed CR protocols and increasing performance techniques (may be with minor modifications).

To satisfy the 5G technology, defined requirement are-

- How to reach the maximum 5G's 1 Tbps data rate threshold when using the CR technology at the access level.

- Better techniques in order to combine the flows coming from multiple access networks.

- How to connect the cognitive radio terminal to the wired networks. This implies new media interfaces definition between the wireless and wired environments.

Cross layer approach [10] for wireless networks like as rapid variations in channel fading, shadowing, mobility etc. Flexible architecture can take advantage of 'good' channel durations. Cross layer approach passes more information across layers. Different cross layer architectures allow more information to flow upward or downward. Figure 2 shows cross layer approach with objective optimization techniques. 


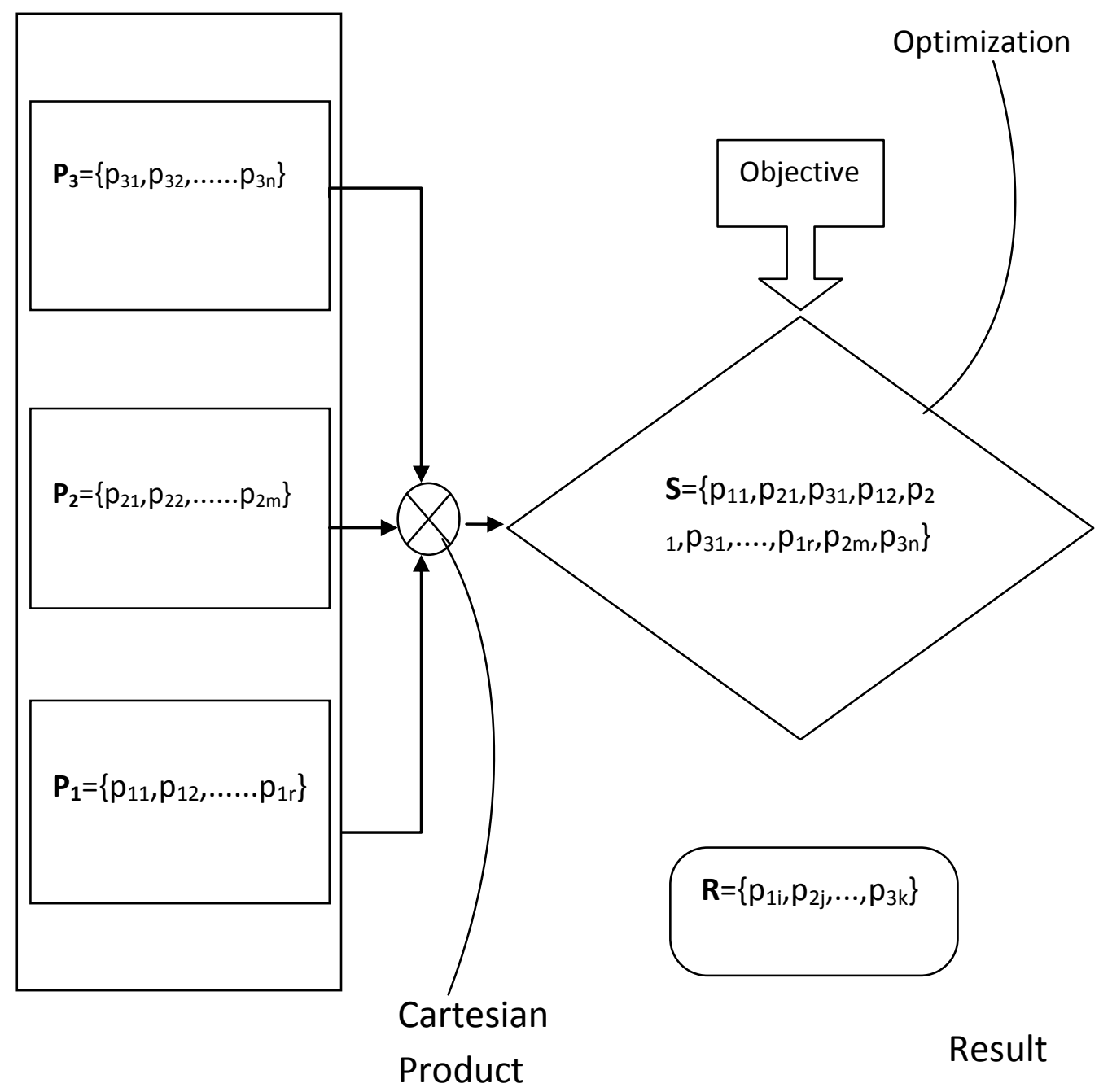

Figure 2: Cross layer approach with objective optimization technique

For better performance in future, Wireless Innovative System for Dynamic Operating Mega communications (WISDOM) is brought into play. It brings unlimited wireless world interconnection, convergence, and cooperation with a large variety of multimedia services at very high data rates. According to wisdom, proposed algorithms monitor the network and resource status and these proposed algorithms are cross layer techniques.

According to cross layer approach, multiple layers and multiple parameters are used. These constraints are defined by cognitive engine according to the environment and other inputs. Different approaches for optimization are-

\section{Single objective optimization}

Find $x^{*}$ which

minimizes $f(x)$

subject to $c_{i} \leq X, i=1,2, \ldots, r$

with $m_{j}(x)=Y, j=1,2, \ldots, h$,

\section{Multiple objective optimizations}

Minimize $F(x)=\left[f_{1}(x), f_{2}(x) \ldots f_{n}(x)\right]$

subject to $c_{i} \leq X, i=1,2, \ldots, r$

with $m_{j}(x)=Y, j=1,2, \ldots, h$,

Quality of Service (QoS) - In order to achieve an efficient quality of service, a lot of methods/algorithms were suggested, involving different disciplines, like single objective function, multiple objective functions mid-step (single objective function but variables from different layers), water filling scheme (for OFDM systems, "sub-carriers are allocated to the users with the best channel quality initially and then adjusted to improve the system performance").

Evolved and extended radio-access solutions are needed to address the above challenges. Extreme mobile broadband is targeting ultra-hotspot scenarios, extreme traffic capacity, end-user throughput, sensor communication, new frequency regimes and spectrum access. 
The most important thing is peak data rate as well as large spectral efficiency that will enable the same $5 \mathrm{G}$ system.

$5 \mathrm{G}$ is single new radio access technology (RAT) that replaces macro cells. It is the combination of present RATs in both licensed and unlicensed bands, plus one or more novel RATs optimized for particular deployments, scenarios and use cases.

Radio Access Technology (RAT) provide a versatile and scalable system concept that supports-

- Higher mobile data volumes (1000x).

- Higher number of connected devices (10x to 100x).
- $\quad$ Higher typical end-user data rates (10x to $100 x)$.

- Longer battery life for low-power connected "machines" (10x).

- $5 x$ lower end-to-end latency with similar overall cost and energy consumption as the networks of today.

In particular, researchers have identified the need for a new RAT for ultra-dense deployments, with the aim of providing a virtual zero latency gigabit experience, used the technique " $5 \mathrm{G}$ hyper service cube" in new radio access technology.

\section{G Hyper Service Cube}

Mobility: 0km/h-500km/h)

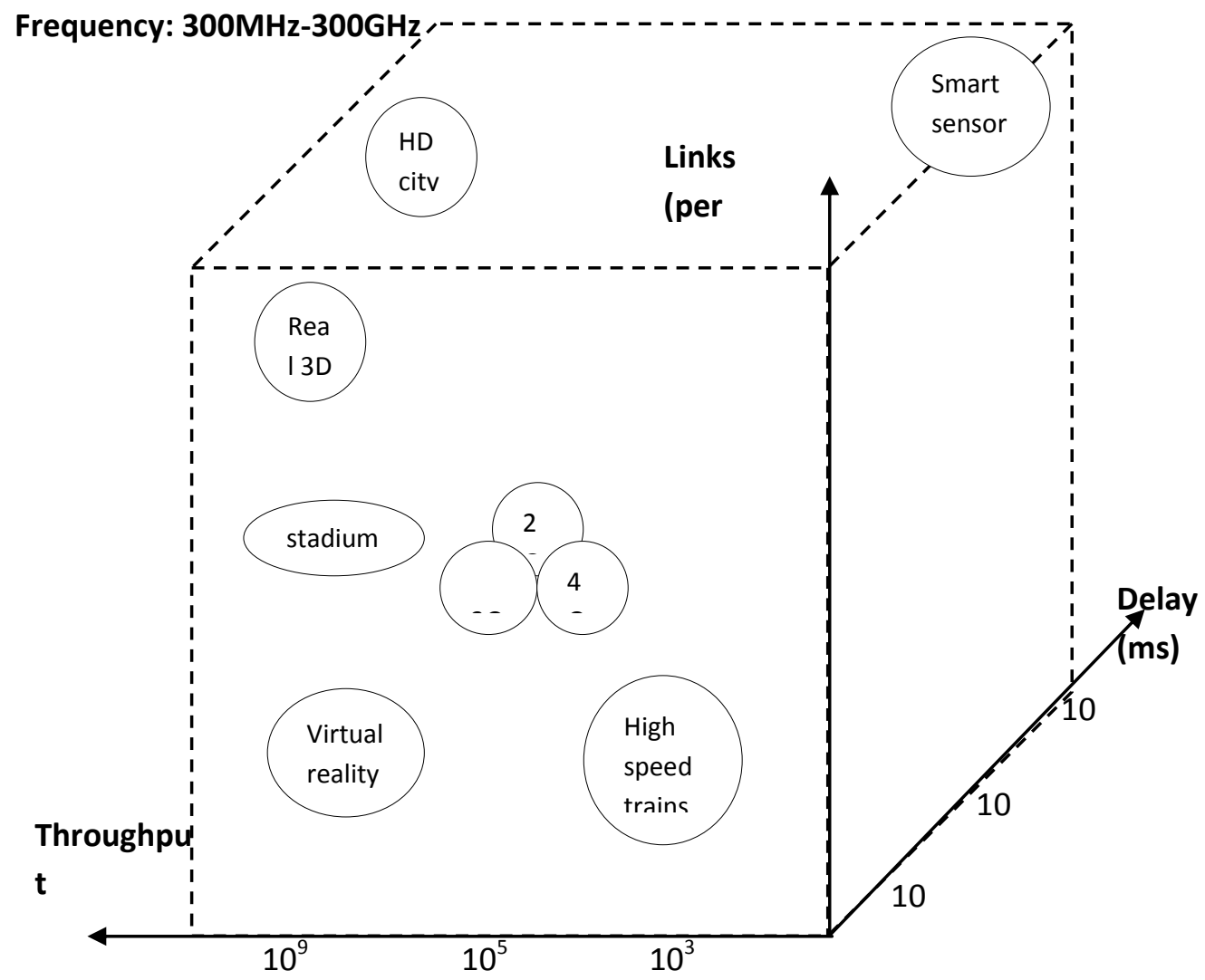

Figure 2: Architectural design of 5G Hyper Service Cube

"5G hyper service cube" gives a multi-dimensional overview in terms of throughput, latency and number of connections required for the many types of services. Figure 3 shows $5 \mathrm{G}$ hyper service cube $5 \mathrm{G}$ networks will need to run

Extreme mobile broadband targeting ultra-hotspot scenarios
- $\quad$ Specific scenarios and use cases

- Sensor communication

- Efficient frequency regimes and spectrum access

- Large amount of traffic capacity / Large amount of multi-Gbps end-user throughput 
New spectrum strategies should be followed like as-

- $\quad$ Licensed shared access (LSA) - The implementation of the LSA concept may take advantage of recent advances in cognitive technology, thus allowing sharing spectrum in a more dynamic way, utilizing frequency, location and time sharing bases.

- Authorized Shared Access (ASA) - To enable timely availability and licensed use of harmonized spectrum for mobile with predictable quality of service.

- Collective Use of Spectrum (CUS) - Allowing spectrum to be used by more than one user simultaneously without requiring a license.

\section{SIMULATION RESULTS}

As per the result from cross layer approach it enhances the performance of distributed cognitive radio network using a multi-objective formulation per cognitive radio node.

Simulation results are drawn for 5G, CR and coalescing 5GCR technology which analyze the BER performance of their respective system. Due to this minimize BER, maximize throughput, minimize power usage and minimize interference with other nodes-

$$
\begin{aligned}
& F(x)=\sum w_{i} f_{i}(x), \quad i \epsilon\{\text { Pe,TP, } P, \text { Int }\} \\
& \text { where } P e \text { is the probability of bit error, } \\
& \text { TP stands for throughput, }
\end{aligned}
$$

$P$ for transmission power and

Int for interference

Figure 4 shows the BER performance analysis of $5^{\text {th }}$ generation. From the simulation result, it can be observed that $5^{\text {th }}$ generation has $10^{\wedge}-4$ BER at an SNR of $26 \mathrm{~dB}$ approx. While, in case of cognitive radio, simulation result shows the improved BER performance. In figure $5, \mathrm{CR}$ has $10^{\wedge}-4$ BER at an SNR of $18 \mathrm{~dB}$ approx. So, CR has approx. $8 \mathrm{~dB}$ performance gain.

Figure 6 shows the BER performance analysis of coalescing $5 \mathrm{G}$ and $\mathrm{CR}$ technology. From the simulation result, it can be observed that combination of $5 \mathrm{G}$ and $\mathrm{CR}$ provides better result than the individuals. The combination 5G-CR has $10^{\wedge}-4$ BER at an SNR of $9 \mathrm{~dB}$ approx. which has best BER results than $5 \mathrm{G}$ and $\mathrm{CR}$.

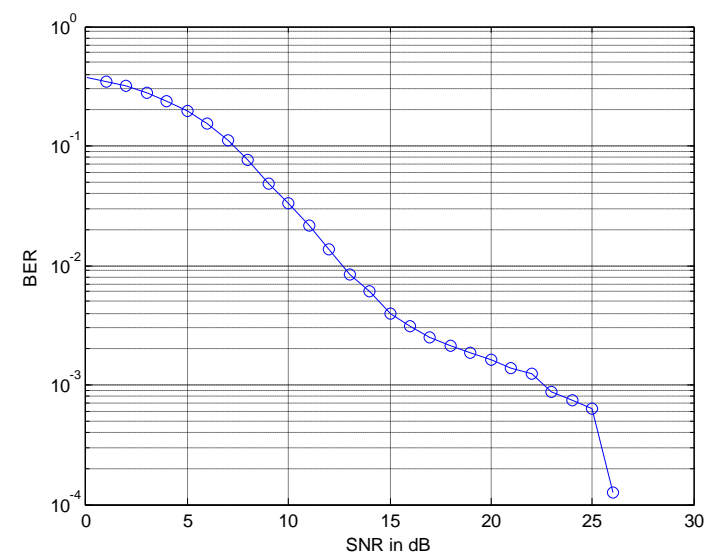

Figure 4: SNR vs. BER for 5G in an indoor environment

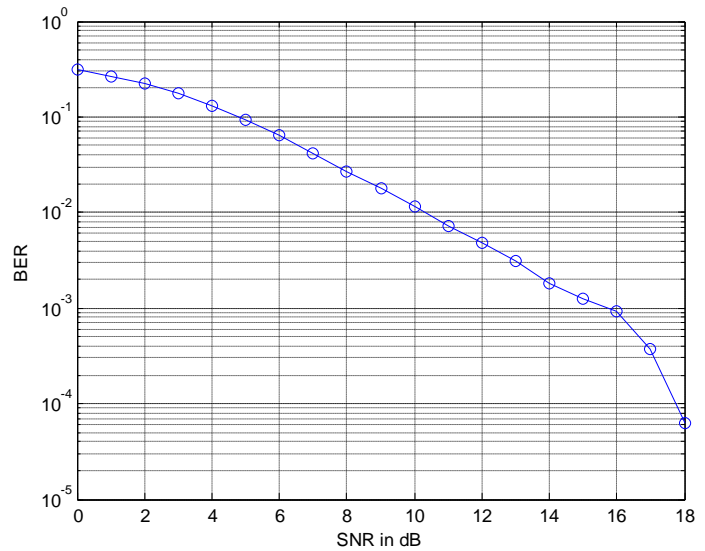

Figure 5: SNR vs. BER for CR technology in an indoor environment

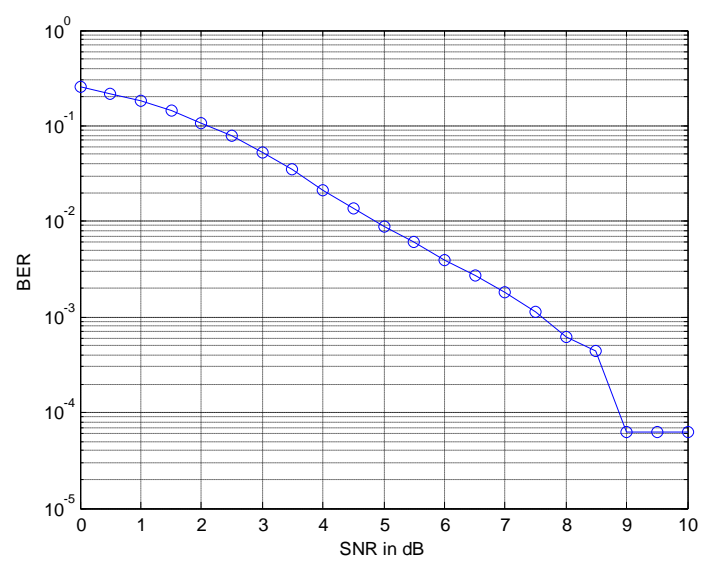

Figure 6: SNR vs. BER for 5G-CR combined technique in an indoor environment

\section{CONCLUSION}

Usage and handling of cross layer design have motivated as a consequence of limitations of layered architectures for a wireless network. Cognitive radio has an inherent relationship with cross layer architecture. Cognitive engine has key role in cross layer optimization of cognitive radio networks. Multiple objective optimizations can be challenging with increase in number of objectives and constraints. Research can be carried out to develop and grow new algorithms in which 5G-CR technology will be used to enhance data rate with low BER and simultaneously, efficient spectrum utilization.

\section{REFERENCES}

[1] Prasad, R., \& Lauridsen, O. M., "Convergence of navigation and communication," in AFCEA International Symposium on Aerospace Technologies and Applications for Dual Use, Rome - 2007.

[2] Prasad, R., \& Ohmori, S., "Ubiquitous easy life," in Second Japan EU Symposium on the Future Internet", Tokyo - 2009.

[3] Prof. R. K. Jain, "Survey of Latest Wireless Cellular Technologies for Enhancement of Spectral Density at Reduced Cost," IJCSI International Journal of Computer Science Issues, Vol. 8, Issue 3, No. 2, May 2011 ISSN (Online): 1694-0814. 
[4] T. Rappaport, Wireless Communications: Principles and Practice, Upper Saddle River, NJ: Prentice Hall, 2002.

[5] Chen, HH, Guizani, M, Mohr, W, "Evolution toward 4G wireless networking," IEEE Network, Volume: 21 Issue1 pp. 4-5, 2007.

[6] Panagiotis Demestichas, Andreas Georgakopoulos, Dimitrios Karvounas, Kostas Tsagkaris, Vera Stavroulaki, Jianmin Lu, Chunshan Xiong, and Jing Yao, " $5 \mathrm{G}$ on the Horizon," in IEEE vehicular technology magazine, pp. 47-53., september 2013.

[7] Cantika Felita and Muhammad Suryanegara, "5G Key Technologies: Identifying Innovation Opportunity," in IEEE, pp. 235-238, 2013

[8] K.C. Chen, R. Prasad, Cognitive Radio Networks, J. Wiley \& Sons, 2009
[9] Prof. Panagiotis Demestichas, "Next Generation Communication Architectures and Technologies," WWRF workshop at ITU-R WP5A, May 2013.

[10] Sanjay Shakkottai, "Cross-layer Design for Wireless Networks," June 23, 2003.

[11] Marek SUCHAŃSKI, "Dynamic Spectrum Management for Military Wireless Networks," 2011.

[12] E. Hossain, D. Miyato and Z. Han, "Dynamic Spectrum Access and Management in Cognitive Radio Networks," Cambridge University Press 2009.

[13] Amir Ghasemi, "Spectrum Sensing in Cognitive Radio Networks: Requirements, Challenges and Design Tradeoffs" IEEE Communications Magazine, April 2008.

[14] Songlin Sun, Yanhong Ju, "Overlay Cognitive Radio OFDM System for 4G Cellular Networks" IEEE Wireless Communications. 